\title{
The Research Characteristics of Traditional Folk Art of Paper Cutting in Modern Society of China
}

\author{
Yang Xiulan, Yang Xiaoyan \\ Shaanxi University of Science and Technology, Xi'an Shaanxi, 710021
}

Keywords: the traditional art of paper cutting; modern society; research characteristics

Abstract: As paper-making was first invented in China, so many British and American paper-cutting artists believe that the earliest art of paper cutting also appeared in China. In fact, the art of paper cutting was proved to own long history in China by a large number of historical materials and poetry through the exploration of its origin. But in recent decades, China's social productive forces have developed rapidly, and a large number of people have poured into the cities. Great changes have taken place in people's way of life. The society has changed from a farming era to a modern time, but China's modern society art of paper cutting and aesthetic value is still attached importance and attention. The traditional art of paper cutting as a symbol of national culture and national art, exploring its characteristics in contemporary research is to outline its historical development, and the realization of a strong witness of the overall understanding.

Traditional paper-cutting is the inheritance of unique expressions and values generated by Chinese people in understanding the natural environment and social life, and contains ancient historical and cultural connotations. The traditional paper cutting is the unique expression and value of our people's understanding of the natural environment and social life. It contains the ancient historical and cultural connotation. Traditional paper cutting is the most common and active art form in folk life. It is an extremely important part of folk art and a basic expression of the survival and inheritance of folk culture, and embodies the feelings, desires, and aspirations of the masses. Paper-cutting is not only a materialized art symbol, but also a symbol of folk culture. Paper-cutting is a kind of folk art form which is closely related to the life of local people in the long-term development of the agricultural society. From the perspective of semiotics, we have re-recognized the art of paper-cutting, and studied the connotation of paper-cutting folk symbols from a deeper level. Now we have re-recognized its development and research characteristics in modern society, and can better inherit the development tradition culture.

\section{The original exploration of the traditional art of paper cutting}

In the "Study on folk art of paper cutting in Northern Shaanxi",Han Liang elaborated the background of the formation,folk resources,stylized artistic features,and cultural skills of folk art of paper cutting,which is the key to interpreting its vision of cultural heritage and local art.Under the influence of primitive culture,primitive philosophy,geographical environment,religious belief and other multicultural factors,the folk art of paper cutting has formed a unified emotional value 
orientation and unique artistic language features.As to the background of the formation of paper cutting,he pursued the source of the paper,thinking that it is the result of the remains of mysterious primitive culture,the amalgamation of traditional original culture,the intersection of farming and grassland ecological culture,as well as the result of the blending of various cultures and religions.If we want to study the native civilization tradition,we must excavate its original culture and the artistic characteristic.This has the unique cultural significance and the historical value to our research.

In the Spring and Autumn Period and the Warring States Period to the Qin and Han Dynasties, there were "cut-out" art form,mainly in the form of decals,Sheng,openwork,which is what scholars call "non-paper cuts.With the improvement of paper-making,paper cutting gradually became popular. From the historical development process,the Tang Dynasty paper-cutting was the most prosperous, and it was already in a period of great development.In Du Fu's poems,there were sentences of "warm water washes my feet,and paper-cutting calls my soul”.The custom of cutting paper and screaming souls has been passed down to the people.Tang Dynasty paper-cutting now housed in the British Museum demonstrate that the art of paper-cutting was already at an advanced level. The complete composition of the picture expresses an ideal state of heaven and earth.Popular in the Tang Dynasty,its scented wood pattern has the characteristics of paper-cutting,such as the "pairs of sheep",the sheep pattern is a typical expression of the art of paper-cut techniques.Tang folk there were also appeared stencil printing plates made by paper-cutting.People used thick paper to sculpt into a flower plate,and the dye was printed on the cloth to form a beautiful pattern.In short,paper-cutting emerged in the Han Dynasty, prevailed in the Tang and song dynasties.In Ming and Qing Dynasties,the art paper cutting began to enter the court,mainly for the rich pattern,meaning and use more widely.Well-preserved and continued to today.This allows us to see the outline of paper-cutting development.

\section{The research performance of traditional folk art of paper cutting in contemporary society}

Chinese traditional art of paper cutting is a rich intangible cultural heritage,which not only displays the ancient charm of Chinese culture,but also greatly enriches the accumulation of Chinese ancient culture.The Chinese traditional art of paper cutting is standing in the treasure house of the world's culture and art,and is a great contribution to the folk art of the world.According to statistics,China's existing thirty ethnic groups have paper-cutting-related customs and traditions. The scope of the long history is rare,and it can be seen that the art of paper cutting has an important position and indelible vitality in people's lives.The traditional art of paper cutting as a symbol of national culture and national art,in contemporary research as follows:

To the Hownet for example,if you search "paper-cutting",there are a total of 6879 results.Among them,there are 1553 folk paper-cuts,1279 the art of paper cutting,696 paper-cutting works,522 People's Republic of China,467 folk crafts,460 folk art,239 intangible cultural heritage,214 folk art,179 Rights Subjects,159 paper-cut teaching Manchu paper-cut 132,artist 121,folk culture 119,Yu County paper-cut 116,paper-cut Animation 103.

Search "paper-cutting embroidery" a total of 182 results,the number of folk paper-cutting papers accounted for 43 at most,folk embroidery accounted for 16.A search for "paper-cutting,clothing" yielded 225 results.Distribution is more even,of which 43 folk paper-cut papers, folk embroidery papers accounted for 16.

The number of research papers is huge and the attention is extremely high.The traditional art of paper cutting has not faded,and it is the backbone of the inheritance and development of national culture and art 


\section{The characteristics of traditional art of paper cutting in contemporary research are}

reflected in the following aspects

First, according to the research classification,it shows that the traditional art of paper cutting is more and more relevant in other fields.The origin and vitality of traditional art of paper cutting is on the rise. For example,there are 14 related categories in the "paper-cutting embroidery"search;15 categories appear in the "paper-cutting" search;in the "paper-cutting clothing"search research paper, there are as many as 30 categories.It can be seen that the traditional art of paper is more and more relevant in other fields.

Second, according to the research theme,the traditional art of paper cutting is more and more prominent in design and application.Searching" paper-cutting application"in the hownet appears 4666 results, showing that people are very concerned about the tradition paper cutting and its application value. Search"the art of paper cutting,design" when a total of 4109 results,the number is also considerable. To sum up,the traditional art of paper cutting elements has received extensive attention in the contemporary.

Third, the results of the study were transmitted to the network,and the art of traditional paper cutting has been unprecedented widespread.The use of the Internet has also brought an objective result, that is, things that people are interested in are more easily found by everyone.People want things to be easy to touch and learn,not only professionally,but also in the interdisciplinary and amateur interests as well as the second class.The development of information technology promotes the wide spread of traditional paper-cutting.

Fourth, the research orientation highlights the practicality,and the results show that the practicality of traditional art of paper cutting is also increasing.

In the North of China,people used to call paper-cuts as "window flowers",pasted on the windows during the New Year holidays to celebrate good luck and good luck.Nowadays,people apply its elements in many fields,such as clothing,packaging design,animation design,etc,and even make bookmarks, borders and advertising stickers.It's nice and practical.

Fifth, the re-understanding and re-summary of the research results is a continuous process of refining. Inheritance and development of the traditional art of paper cutting is also a bridge to link it with modern life.

In the "analysis of the modern transformation of Folk art of paper cutting form",Zhang Lin mentioned that "Chinese Folk art of paper cutting began to transform in the 1940s,such as Lu Yi and $\mathrm{Gu}$ Yuan of Yan'an created a themed paper-cut works with anti-Japanese colors through a new paper-cutting process. The creation of this work made folk art of paper cutting not only confined to the expression of good feelings,but also introduced this theme into it for thematic creation."In addition, she referred to the three characteristics of the folk art paper cutting form,One is the two-dimensionality of paper-cutting,that is,the spatial flatness; the second is the intentionality of modeling; the third is the symbolicity of image. These three characteristics enhance the artistry of paper-cutting works

With regard to the the inheritance and development of folk art of paper cutting, data showing that there are art of paper cutting education in some areas and schools, and more and more professionals have made great efforts in inheritance. The art of paper cutting has been improved, and it has become closer to modern life in aesthetics and decoration, especially the emergence of derivatives of art of paper cutting. It is a good bridge for paper-cutting into modern life.

Sixth, using modern research methods such as databases and literature libraries,we have learned that a new development model of "traditional art of paper cutting+materials+technology"is currently being formed.

The ancient folk art of paper cutting mainly relies on manual operation, modern technology has 
improved,and the production of the machine is also hand-made,and the paper-cutting is more exquisite.In the production of New Year's greeting cards,paper-cutting has evolved from two-dimensional to three-dimensional.In terms of materials,solid red paper and scissors were used in the past,and even knives were used.Color paper-cutting appeared in the modern,and the materials were rich and varied,and they were no longer limited.Through research,it is not difficult to find that it is gradually forming a new development model,with intaglio and positive engraving,and the layering is very rich

\section{Conclusion}

Compared with the cultural customs and cultural connotations of the Tang Dynasty traditional paper-cutting,modern research direction is more inclined to the traditional art of paper cutting the art part.The function of the cultural part becomes weak with the large population flowing into the city.However,paper-cutting is still loved by people and folk artists all over the world.The traditional art of paper cutting has not been abandoned by people.A series of data indicated that the research characteristics of the traditional art of paper cutting in modern society will change with the changes of technology,custom,living environment,aesthetic pursuit,social popular trend,material,design and development in many fields.It's moving towards convergence,towards modernity.Under the promotion of modern information technology,the traditional folk art of paper cutting has also been fully developed.

\section{References}

[1] Huo Shaofeng.Analysis on the Development of the Paper-cutting and Existing Problems of Liyang Paper-cutting[J]. Drama House, 2014(10): 221.

[2]Zheng Ling. Aesthetic Characteristics of Folk Paper-cut Pattern Symbols in Shaanxi Province[J]. Art Research, 2008(01): 48-49.

[3] Jia Danni. The Artistic Features and Protection of Folk Paper-cutting in Shaanxi Province [J]. Weinan Normal College, 2018, 33(15): 76-81.

[4] Zhao Mingnan. Stuffed flowers [J]. Culture Monthly. 2015(02)

[5] Dan Ruo. Paper-cutting dreams [J]. Western Development. 2009 (11)

[6] Liang Xin. Reflections on the Teaching of Paper-cut Patterns in Northern Shaanxi[J].Reading the World.2016(20)

[7] Zhang Xuesong; Viewing Jian'an and Sheng Tang Feng Gu from the Perspective of Image Construction [J]; Journal of Beijing Normal University (Humanities and Social Sciences);1999 05

[8] Kang Zhen; The Process of Poetic History in the Vision of Cultural Integration_-On the Cultural History Significance of Sui Dynasty Poetry[J];Journal of Beijing Normal University(Social Science Edition);2005-03

[9]Zheng Weishan. Talking about Chinese Folk Paper-cut Art[J]. Decoration, 2003, (10) :32.

[10] Zhang Qun. Modern Transformation and Application Research of Paper-cut Art [D]. Beijing: Beijing Forestry University, 2011

[11] Gao Hui. Inheritance and Development of Chinese Folk Paper-cut Art[J]. Journal of Anshun University, 2014,16 (02): 22-23.

[12] Dou Qiangchao. On the Modern Deduction of Chinese Traditional Folk Paper-cut Art[J]. Journal of Chengde National Teachers College, 2010, 30(01): 51-54. 